\title{
Die Entropie des Sinns in einer Welt voller Gier
}

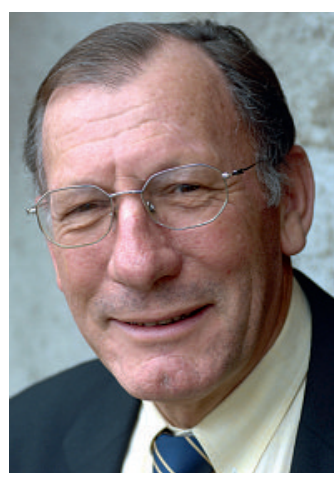

Jean Martin
«Assistance publique - Hôpitaux de Paris», der eine grosse Reihe öffentlicher und Universitätsspitäler in der Region Paris angeschlossen ist, hat seit 1995 eine sehr aktive Abteilung für ethische Fragen. Dort habe ich im Mai an einem Seminar mit hochkarätigen Rednern teilgenommen (was zeigt, dass die Angelsachsen hier nicht die alleinige Führung innehaben ...), wie beispielsweise Elisabeth Sledziewski von der Universität Robert Schuman aus Strassburg. In ihrem Beitrag führte die Rednerin aus, wie es der Politik - dem Leben in der Gesellschaft - an einem wahren Sinn fehlt. Sinn fällt aber nicht vom Himmel, Sinn entsteht, entwickelt sich und kann nicht als «Reserve» eingelagert werden für eine Zeit wie die heutige, die ihre wegweisenden Orientierungspunkte aus den Augen verloren hat. Die Entropie des Sinns?

Sledziewski bezieht sich auf den Nobelpreisträger für Wirtschaftswissenschaften aus dem Jahr 2001, Joseph Stiglitz, einen vehementen Kritiker der aktuellen Entwicklungen, insbesondere des Finanzsystems und auf sein neustes Buch mit dem Titel Freefall, in deutscher Übersetzung Im freien Fall [1]. Das Streben nach materiellem Erfolg um jeden Preis führt zu einer eklatanten «Marketization» unseres Lebens.

Das System basiert auf Gier - Schwindel und Betrug sind die Wurzeln allen Tuns (!), so Sledziewski. Es zählen allein die Interessen einzelner Wirtschaftsgruppen, wir erleben eine Tragödie des Allgemeinguts (Tragedy of the Commons), wie Garrett Hardin sie in seinem Buch mit dem prophetischen Titel Gefährdete Zukunft aus dem Jahr 1968 beschreibt. Der Individualismus verdrängt die Allmende, den Gemeinschaftsbesitz unserer bäuerlichen Vorfahren. In der Ausgabe des französischen Wochenmagazins Le Nouvel Observateur vom 20. Januar schreibt Russell Banks, die USA strebe seit einem Jahrhundert nach einer Plutokratie, einer Herrschaft durch die Vermögenden, und stehe nun kurz vor ihrem Ziel. Und die Schweiz nimmt sich ein Beispiel, beobachtet man nur den zunehmenden Einfluss des Geldes auf Parteien und Politik. Dabei ist kaum vorstellbar, dass die heute omnipräsenten Wirtschaftslobbys, einschliesslich der Krankenversicherer, tatsächlich zu einer besseren Demokratie beitragen. Zwischen Macht und Sinn tut sich eine Kluft auf.

$\mathrm{Zu}$ den Hoffnungen, die in den amerikanischen Präsidenten Obama gesetzt wurden, sagt ein Beobachter: «Er hat nur die Sessel auf der Brücke der Titanic leicht verrückt.» Die depressive Stimmung in der Wirtschaft wirkt sich auf alle gesellschaftlichen Bereiche aus, auch auf das Gesundheitssystem. Viele haben in der heutigen Zeit die Richtung verloren [2].

Eine unangemessene und bürokratische Reaktion auf diesen Verlust des Sinns und des Bewusstseins für das Allgemeinwohl ist ein übertriebener legislativer
Eifer. Weil es an Werten mangelt, werden Gesetze erlassen (ich habe es jüngst für den medizinischen Bereich beschrieben [3]). Während Montesquieu postuliert, die Seltenheit und Majestät der Gesetze zu bewahren, wird mehr und mehr über gesetzliche Regelungen gesteuert und das Gesetz wird zunehmend pointillistisch.

Wir leben in der Zeit des homo consumator, des homo eligens, der nicht nur die Möglichkeit hat zu wählen, sondern dessen Existenz sich nur noch in der stetigen Suche begreift, «etwas anderes» zu kaufen oder zu tun. Häufig hat der Erhalt der Wahlmöglichkeit viel grössere, existentielle Bedeutung als die Dinge, zwischen denen wir wählen sollen - und die immer rascher ihre Wertigkeit verlieren.

Das in Freiburg veröffentlichte Magazin La Revue Durable bringt Analysen über ökologische Themen und verdient durchaus stärkere Beachtung. In der Ende 2009 erschienenen Ausgabe Nr. 36 liest man ein Interview mit Olivier Abel, Professor für Philosophie am Institut protestant in Paris [4]. Zu der von Max Weber als protestantisch beschriebenen Ethik sagt Abel, im Zentrum unserer Anthropologie stehe viel zu sehr der arbeitende, Leistung erbringende Mensch. Dabei sei Kreativität aber schönes Beiwerk, von wesentlicher Bedeutung seien dahingegen die Werte des Zusammenlebens. Absolut entscheidend sei eine Veränderung der Gewohnheiten in Richtung eines von einfacher, schlichter Grosszügigkeit geprägten Miteinanders. Er würde streben nach der Abkehr vom zentralen Paradigma, in dem die Schweiz und Europa ihre Zukunftschancen sehen - Wissensgesellschaft, primäre Investition von Ressourcen in die Forschung - und für eine Gegenbewegung. Für viele ist es eine Häresie.

Zurück zum Seminar. Wollten wir unserem Tun wieder Sinn geben, so sei dies nur durch die Öffnung gegenüber dem anderen möglich, betonte Sledziewski, z. B. anerkennen, dass wir unserem Nächsten, in beruflichen und persönlichen Beziehungen und in der Beziehung zwischen Pflegendem und Kranken, unserer Gemeinschaft und der gesamten Menschheit gegenüber in der Pflicht stehen. Jede Ethik ist ein Vertrag, knüpft ein Band zwischen uns und dem anderen. Dies gilt gleichermassen für das so vernachlässigte Familienleben: Wie viele von uns scheinen der Ansicht zu sein, dass Familienbande eine lose, nicht verpflichtende Verbindung zwischen den Mitgliedern sind! Stattdessen gilt aber auch heute, je schutzbedürftiger eine Person ist, desto mehr sind wir ihr verpflichtet, so haben wir es uns in der Präambel unserer Bundesverfassung von 1999 zum Grundsatz gemacht: «gewiss ... dass die Stärke des Volkes sich misst am Wohl der Schwachen».

Jean Martin, Mitglied der SÄZ-Redaktion und der Nationalen Ethikkommission Dezember 2009; Nr. 36, S. 9-13. 